\title{
Research on Correlation between TCM Syndrome Distribution Characteristics and Prognosis of Hypertensive Intracerebral Hemorrhage Operation
}

\author{
Gang Yang1*, Jianju Feng², Shaojun Yang1, Junjie Lv¹, Donghai Yuan'1, Chenbing Wang1, \\ Feng Ding ${ }^{1}$, Chao Gu${ }^{1}$, Gaofeng Shao ${ }^{1}$ \\ ${ }^{1}$ Department of Neurosurgery, Zhuji Affiliated Hospital of Shaoxing University, Shaoxing, China \\ ${ }^{2}$ Radiology Department, Zhuji Affiliated Hospital of Shaoxing University, Shaoxing, China \\ Email: *tekeyang@sina.com
}

How to cite this paper: Yang, G., Feng, J.J., Yang, S.J., Lv, J.J., Yuan, D.H., Wang, C.B., Ding, F., Gu, C. and Shao, G.F. (2021) Research on Correlation between TCM Syndrome Distribution Characteristics and Prognosis of Hypertensive Intracerebral Hemorrhage Operation. Journal of Biosciences and Medicines, 9, 138-144.

https://doi.org/10.4236/jbm.2021.94011

Received: March 23, 2021

Accepted: April 20, 2021

Published: April 23, 2021

Copyright $\odot 2021$ by author(s) and Scientific Research Publishing Inc. This work is licensed under the Creative Commons Attribution International License (CC BY 4.0).

http://creativecommons.org/licenses/by/4.0/

\begin{abstract}
Objective: This study is to analyze correlation between traditional Chinese medicine (TCM) syndrome distribution characteristics and prognosis of hypertensive intracerebral hemorrhage (HICH) operation. Methods: In this study, a total of 150 patients who had received HICH operation from April, 2017 to December, 2020 in our hospital and conformed to inclusion standards were selected. According to classification of TCM syndromes, amount of bleeding of patients was recorded through multiple radiological technologies, baseline information was collected, and prognosis was investigated. The final event was long-term follow-up visit of all-cause mortalities. Moreover, correlation between prognosis and TCM syndromes was analyzed. Results: It found through investigations that there are no statistically significant differences in composition ratio of TCM syndromes among patients with different genders and different age ranges $(P>0.05)$. Among so many TCM syndromes, bleeding amount of patients with declining vitality and distraction is the highest, while bleeding amount of patients with stirring wind due to yin deficiency is the lowest. With respect NIHSS scores, the patients with declining vitality and distraction show the highest NIHSS scores at admission and $10 \mathrm{~d}$ of the course of the disease, followed by patients with upward disturbance of wind-fire. There are statistically significant differences among these two groups $(P<0.05)$. In this study, follow-up visits are performed to all 150 patients and the average follow-up visit time ranges within $2-15$ months. A total of 13 deaths are reported. Number of all-cause deaths has statistically significant differences among different syndromes $(P<0.05)$. Conclusions: Evolutionary characteristics of TCM syndromes of HICH are manifested by
\end{abstract}


development from evidence-based symptoms to deficiency syndromes. Different syndromes are correlated with prognosis of patients. On the one hand, this can be used as an objective index for TCM syndrome classification. On the other hand, this is conducive to judge prognosis recovery of patients and to apply the corresponding symptomatic treatment.

\section{Keywords}

HICH, TCM Syndromes, Distribution Characteristics, Prognosis, Correlation

\section{Introduction}

Hypertensive intracerebral hemorrhage ( $\mathrm{HICH})$ generally refers to patients who have spontaneous Intracerebral Hemorrhage combined with hypertension. It is characteristic of relatively high morbidity, disability rate and fatality, and it is a great threat to physical health and quality of life of patients [1]. Currently, operation is still an effective treatment to HICH. Researches on prognosis of patients with HICH attract key attentions in clinics. However, correlation between distribution characteristics of TCM syndromes and prognosis of patients with HICH still remains unknown [2] [3]. In this study, 150 patients who received HICH operation from April, 2017 to December, 2020 in our hospital and conformed to inclusion criteria were selected and classified according to TCM syndromes. Whether there's a correlation between distribution characteristics of TCM syndromes and prognosis of patients was determined through a statistical analysis of clinical data. Results were conducive to make reasonable prognosis of patients after assessment and provide new ideas and theories for treatment of $\mathrm{HICH}$. Results are introduced in the following text.

\section{Data and Methodology}

\subsection{General Information}

A total of 150 patients who received $\mathrm{HICH}$ operation from April, 2017 to December, 2020 in our hospital and conformed to inclusion criteria were reviewed. Among them, there were 89 males and 61 females. They aged between $41-85$, averaging at $(77.84 \pm 1.52)$. All patients had hypertension for 5 - 18 years, (11.75 $\pm 1.85)$ years in average.

\subsubsection{Inclusion Criteria}

Patients who meet following items were included: 1) Chinese Guidelines for Diagnosis and Treatment of Cerebral Hemorrhage [4] in 2015 and Experts' Consensus on Chinese Traditional Medicine and Western Medicine Combined Diagnosis and Treatment of Hypertensive Intracerebral Hemorrhage in Acute Stage in 2016 [5], Stroke Diagnosis and Assessment Criteria of Curative Effect (Trial) in Traditional Chinese Medicine [6], accompanied with evident history of hypertension; 2) scores of Glasgow Coma Scale [7] (GCS) $\geq 8$; 3) Volunteered to 
join in the experiment and signed the agreement; 4) approved by Ethics Committee of the hospital.

\subsubsection{Exclusion Criteria}

Patients who meet one of following items were excluded: 1) intracerebral hemorrhage caused by other brain diseases or trauma; 2) patients combined with surgical contraindications; 3 ) patients with cerebrovascular malformation.

\subsection{Methodology}

\subsubsection{Acquisition of General Clinical Data}

Baseline data of all patients were collected, including name, age, gender, admission number $(\mathrm{AD})$, etc. Relevant clinical data were collected, including disease status at admission, duration from morbidity to admission, brain CT report and bleeding amount at admission, and clinical National Institute of Health stroke scale (NIHSS) [8]. If NIHSS $<4$, patients were diagnosed as mild neurologic impairment. If NIHSS is between $4-15$, patients were diagnosed as moderate impairment. If NIHSS $>15$, patients were diagnosed sever impairment. All patients were assessed by professional physicians at admission and $10 \mathrm{~d}$ of the course of the disease.

\subsubsection{Follow-up Visit and Judgment of Stroke Outcome}

In this study, follow-up visit started from the first diagnosis as stroke to all-cause death. All-cause death includes cardiovascular death and deaths for other reasons. Finally, patients were confirmed by senior neurological physicians with rich experiences according to radiological and direct medical proofs. Medical documents and certificate of deaths of died patients were checked.

\subsection{Statistical Processing}

All collected data were analyzed and processed by SPSS19.0. Enumeration data were expressed by percentage and checked by Chi-square test. Measurement data were expressed by $\bar{\chi} \pm s$ and checked by t-test. $P<0.05$ indicates statistically significant differences.

\section{Results}

\subsection{Effects of Demographic Characteristics on Distribution Characteristics of TCM Syndromes}

According to investigation, there's no statistically significant difference in distribution characteristics of TCM syndrome among patients with different genders and different age ranges $(P>0.05)$. Results are shown in Table 1.

\subsection{Comparison of Bleeding amount and NIHSS Score among Different Syndromes}

Among so many TCM syndromes, patients with declining vitality and distraction show the highest bleeding amount, while patients with stirring wind due to 
Table 1. Effects of demographic characteristics on distribution characteristics of TCM syndromes [n (\%)].

\begin{tabular}{|c|c|c|c|c|c|c|c|c|}
\hline & Data & $\begin{array}{c}\text { Number } \\
\text { of cases }\end{array}$ & $\begin{array}{c}\text { Closed Heart } \\
\text { due to } \\
\text { phlegm-heat }\end{array}$ & $\begin{array}{c}\text { Qi deficiency } \\
\text { and blood stasis }\end{array}$ & $\begin{array}{c}\text { Closed mind } \\
\text { due to phlegmatic } \\
\text { hygrosis }\end{array}$ & $\begin{array}{l}\text { Declining } \\
\text { vitality and } \\
\text { distraction }\end{array}$ & $\begin{array}{l}\text { Upward } \\
\text { disturbance } \\
\text { of wind-fire }\end{array}$ & $\begin{array}{c}\text { Stirring } \\
\text { wind due to } \\
\text { yin deficiency }\end{array}$ \\
\hline \multirow{2}{*}{ Gender } & Males & 89 & $18(20.22)$ & $14(15.73)$ & $28(31.46)$ & $13(14.61)$ & $10(11.24)$ & $6(6.74)$ \\
\hline & Female & 61 & $14(22.95)$ & $7(11.48)$ & $20(32.79)$ & $11(18.03)$ & $5(8.20)$ & $4(6.56)$ \\
\hline \multirow{4}{*}{ Age } & $40-49$ & 24 & $4(16.67)$ & $3(12.50)$ & $6(25.00)$ & $7(29.17)$ & $2(8.33)$ & $2(8.33)$ \\
\hline & $50-59$ & 32 & $7(21.88)$ & $5(15.63)$ & $10(31.25)$ & $5(15.63)$ & $3(9.38)$ & $2(6.25)$ \\
\hline & $60-69$ & 58 & $12(20.69)$ & $6(10.34)$ & $19(32.76)$ & $8(13.79)$ & $8(13.79)$ & $5(8.62)$ \\
\hline & $>70$ & 36 & $9(25.00)$ & $7(19.44)$ & $13(36.11)$ & $4(11.11)$ & $2(5.56)$ & $1(2.78)$ \\
\hline
\end{tabular}

yin deficiency show the lowest bleeding amount. In term of NIHSS score, patients with declining vitality and distraction achieve the highest NIHSS score at admission and $10 \mathrm{~d}$ of the course of the disease, followed by patients with upward disturbance of wind-fire. There are statistically significant differences between these two syndromes in term of NIHSS score $(P<0.05)$. Results are shown in Table 2.

\subsection{Comparison of Follow-up Visit Time and All-Cause Deaths among Different Syndromes}

Follow-up visits were performed to all 150 patients. The average follow-up visit time ranged between 2 - 15 months, and 13 deaths were reported. There are statistically significant differences among different syndromes in term of number of all-cause deaths $(P<0.05)$ (Table 3$)$.

\section{Discussions}

$\mathrm{HICH}$ is a serious complication of hypertension and it has high morbidity in the group aged between 50 - 70 [9]. With the intensifying aging degree of national residents, number of patients with $\mathrm{HICH}$ is increasing continuously. People suffer $\mathrm{HICH}$ suddenly and the disease worsens quickly, accompanied with high disability rate and fatality rate [10]. Therefore, disclosing the correlation between distribution characteristics of TCM syndromes and prognosis of patients has important clinical significance to explore new ideas for treatment to $\mathrm{HICH}$. TCM believes [11] that HICH belongs to the scope of "stroke" and TCM has accumulated abundant experiences in treatment to stroke. Moreover, the theory of "abnormal flow of the blood is extravasated blood" was proposed, which advocated simultaneous address both symptoms and root causes. However, key attentions shall be paid to eliminating symptoms in the acute period. Nowadays, $\mathrm{HICH}$ still lacks of evidence-based medicine. Therefore, this study focused on patients after $\mathrm{HICH}$ operation.

According to survey results, patients with closed mind due to phlegmatic hygrosis accounted for the highest proportion $(32.00 \%, 48 / 150)$ among the selected 150 cases, followed by patients with closed Heart due to phlegm-heat $(21.33 \%$, 
Table 2. Comparison of bleeding amount and NIHSS score among different syndromes at admission.

\begin{tabular}{ccccc}
\hline \multirow{2}{*}{ TCM syndromes } & $\begin{array}{c}\text { Number } \\
\text { of cases }\end{array}$ & Bleeding amount & \multicolumn{2}{c}{ NIHSS } \\
\cline { 3 - 5 } & & & Admission & $10 \mathrm{~d}$ \\
\hline Closed Heart due to phlegm-heat & 32 & $56.21 \pm 13.25$ & $36.73 \pm 1.98$ & $21.21 \pm 13.28$ \\
Qi deficiency and blood stasis & 21 & $45.12 \pm 12.46$ & $32.26 \pm 3.16$ & $16.12 \pm 12.32$ \\
Closed mind due to phlegmatic hygrosis & 48 & $34.45 \pm 13.37$ & $25.73 \pm 2.98$ & $14.21 \pm 13.28$ \\
Declining vitality and distraction & 24 & $66.67 \pm 12.26$ & $49.26 \pm 3.18$ & $32.12 \pm 12.32$ \\
Upward disturbance of wind-fire & 15 & $48.16 \pm 13.28$ & $41.73 \pm 2.96$ & $28.21 \pm 13.28$ \\
Stirring wind due to yin deficiency & 10 & $33.35 \pm 12.41$ & $19.26 \pm 1.17$ & $12.12 \pm 12.32$ \\
\hline
\end{tabular}

Table 3. Comparison of follow-up visit time and all-cause deaths among different syndromes [n (\%)].

\begin{tabular}{cccc}
\hline TCM syndromes & Number of cases & $\begin{array}{c}\text { Follow-up visit } \\
\text { time (months) }\end{array}$ & All-cause deaths \\
\hline Closed Heart due to phlegm-heat & 32 & $2-15$ & $2(6.25)$ \\
Qi deficiency and blood stasis & 21 & $2-14$ & $1(4.76)$ \\
Closed mind due to phlegmatic hygrosis & 48 & $2-14$ & $1(2.08)$ \\
Declining vitality and distraction & 24 & $3-15$ & $5(20.83)$ \\
Upward disturbance of wind-fire & 15 & $2-13$ & $4(26.67)$ \\
Stirring wind due to yin deficiency & 10 & $3-14$ & $0(0.00)$ \\
\hline
\end{tabular}

32/150). Both of these two syndromes have a major symptom of coma. This might be because intracranial pressure increases quickly due to the high bleeding amount of patients after morbidity and the neurological functions affected and lost for temporary. Additionally, patients with declining vitality and distraction accounts for a relatively high proportion after $\mathrm{HICH}$ operation, which might be related with vital impairment and unsmooth air flow in organs after long time in bed [12]. Besides, effects of gender and age on TCM syndromes of patients with $\mathrm{HICH}$ were investigated, finding no evident influences. However, the morbidity rate of $\mathrm{HICH}$ reaches the peak in the age group of $60-69$, which might be interpreted by cerebral arteriosclerosis under long-term hypertension in the old [13] [14]. Effects of bleeding amount and NIHSS score on TCM syndromes of patients with $\mathrm{HICH}$ were further investigated. It found that patients with declining vitality and distraction show the highest bleeding amount, while patients with stirring wind due to yin deficiency show the lowest bleeding amount. Moreover, patients with declining vitality and distraction present the highest NIHSS score at admission and $10 \mathrm{~d}$ of the course of the disease, followed by patients with upward disturbance of wind-fire. There are statistically significant differences between these two syndromes in term of NIHSS score $(P<0.05)$. Obviously, disease conditions of patients with declining vitality and distraction and patients with upward disturbance of wind-fire are relatively complicated and serious, 
who deserve high clinical attentions. In prognosis follow-up visits, 13 deaths were reported. There are statistically significant differences among different syndromes in view of all-cause deaths $(P<0.05)$. This reveals that TCM syndromes are complicated, changing and personalized in a series of processes from onset to development. Judging syndrome characteristics of patents timely and accurately and mastering their evolutionary laws are vital to correct treatment based on syndromes and full development of strong advantages of TCM in HICH treatment [15].

\section{Conclusion}

To sum up, TCM syndromes of HICH develop from evidence-based symptoms to deficiency syndromes. Syndromes are related with disease condition and prognosis of patients. On the one hand, this can be an objective indicator of TCM syndrome classification. On the other hand, this is beneficial to judge prognosis recovery of patents and adopt corresponding symptomatic treatment. However, this study involves a small sample size and the follow-up visit was limited within 1 year after the operation, resulting in unconvincing conclusions. Future studies are considered to expand sample size and prolong follow-up visit period.

\section{Fund-Supported Projects}

Zhejiang Medical Association Clinical research fund projects 2019ZYC-A157. Shaoxing Health Science and technology plan 2017CX025.

Zhejiang Health Development Foundation 2019ZD059.

\section{Conflicts of Interest}

The authors declare no conflicts of interest regarding the publication of this paper.

\section{References}

[1] Yang, B., Liang, Q.H., Xiong, X.G., et al. (2014) A Comparative Research on Proteomics of Liver Yang Syndrome in Acute Stage and Stirring Wind Due to Yin Deficiency in Recovery Stage of HICH. Journal of Hunan University of Chinese Medicine, 34, 34-38, 65.

[2] Xiang, M.J. (2014) Research on Relations of TCM Syndromes of HICH with Content of Serum C-Reactive Protein and Integration of Neurologic Impairment. $\mathrm{He}$ nan Traditional Chinese Medicine, 34, 1495-1496.

[3] Tang, W. and Li, Q. (2013) Relationship between TCM Syndromes and Serum Ischemia-Modified Protein Level in Patients with Acute Intracerebral Hemorrhage. Chinese Journal of Traditional Chinese Medicine Information, 20, 17-18.

[4] Wei, P., You, C., Jin, H., et al. (2014) Correlation between Serum IL-1beta Levels and Cerebral Edema Extent in a Hypertensive Intracerebral Hemorrhage Rat Model. Neurological Research: An Interdisciplinary Quarterly Journal, 36, 170-175. https://doi.org/10.1179/1743132813Y.0000000292

[5] Li, X.W., Sun, Z.S., Zhao, W.M., et al. (2013) Effect of Acetylsalicylic Acid Usage 
and Platelet Transfusion on Postoperative Hemorrhage and Activities of Daily Living in Patients with Acute Intracerebral Hemorrhage. Journal of Neurosurgery, 118, 94-103. https://doi.org/10.3171/2012.9.JNS112286

[6] Xie, Y.G. (2019) Effects of Acupuncture in TCM on Neurological Function, Self-Care Ability and Quality of Life of Patients after Intracerebral Hemorrhage Operation. Journal of Guangzhou Medical University, 47, 92-94.

[7] Tapia-Pérez, J.H., Gehring, S., Zilke, R., et al. (2014) Effect of Increased Glucose Levels on Short-Term Outcome in Hypertensive Spontaneous Intracerebral Hemorrhage. Clinical Neurology and Neurosurgery, 118, 37-43. https://doi.org/10.1016/j.clineuro.2013.12.018

[8] Feng, Z.Q., Zhao, X.P., Fan, X.X., et al. (2016) Research on the Relationship between TCM Constitution and First Syndrome in Acute Stage of Patients with Intracerebral Hemorrhage. Practical Clinical Journal of Integrated Traditional Chinese and Western Medicines, 16, 23-25.

[9] Cui, C.L. and Wang, Z.Q. (2016) Multi-Factor Logistic Regression Analysis on Prognosis of Intracerebral Hemorrhage. Journal of Emergency in Traditional Chinese Medicine, 25, 1037-1038, 1073.

[10] Wang, S.Z. and Jia, C.H. (2016) Research on Endogenous Wind of Stroke in Traditional Chinese Medicine Based on Comparison between Traditional Chinese Medicine and Western Medicine. World Chinese Medicine, 11, 614-617.

[11] Li, Z.J., Shao, H.M., Zhang, D.D., et al. (2014) Research on TCM Syndrome Factors and Syndrome Distribution Laws in Acute Stage of Intracebral Hemorrhage. Journal of Emergency in Traditional Chinese Medicine, 23, 2047-2048.

[12] Chen, G.C., Zhang, G.M., Luo, Y.R., et al. (2015) Application of Integrated Traditional Chinese Medicine and Western Medicine in Activating Blood Circulation of Patients with Intracerebral Hemorrhage. Journal of Integrated Chinese and Western Medicine Cardio-Cerebrovascular Disease, 13, 1782-1783, 1808.

[13] Li, X.L., Wang, Z.Y., Sun, S.Z., et al. (2017) Syndrome Differentiation and Treatment in Chinese Medicine: Curative Effect of Obstruction-Removing Therapy on Acute Stable Type of Intracerebral Hemorrhage. Shanxi Medical Journal, 46, 529-530.

[14] Dai, F.Y., Wang, Y., Yu, X.L., et al. (2015) Logistic Regression Analysis of TCM Syndromes of Intracerebral Hemorrhage and Influencing Factors of Good Prognosis. Journal of Zhejiang University of Chinese Medicine, 14, 115-119.

[15] Zhang, X., Liu, Y.X., Yan, L., et al. (2015) Analysis on Five Elements' Motion and Six Kinds of Natural Factors from the Date of Birth of 332 Patients with Intracerebral Hemorrhage. World Science and Technology-Modernization of Traditional Chinese Medicine, 17, 2547-2552. 